\title{
Franz-Keldysh Effect in Semiconductor T-Wire in Applied Magnetic Field
}

\author{
Justino R. Madureira, Marcelo Z. Maialle ${ }^{\dagger}$, and Marcos H. Degani ${ }^{\ddagger}$ \\ *Instituto de Física, Universidade Estadual de Campinas, CP 6165, 13083-970, Campinas-SP, Brazil \\ ${ }^{\dagger}$ Liceu Vivere, Rua Duque de Caxias Norte, 550, 13635-000, Pirassununga-SP, Brazil \\ ${ }^{\ddagger}$ Haras Degani, Ave Fioravante Piovani 1000, 13250-000, Itatiba-SP, Brazil
}

Received on 31 March, 2003

\begin{abstract}
We have calculated the optical absorption spectra of magnetoexcitons in T-wire semiconductor. It is calculated using the semiconductor Bloch equations in time and real-space domains. The peak and the linewitdth of the fundamental exciton transition are investigated as a function of the applied magnetic and static electric field. The latter is applied along the wire axis and induces a broadening in the absorption spectra line, energy shift, and the characterisitic Franz-Keldysh oscillations. The exciton binding energy enhances increasing the magnetic field strength.
\end{abstract}

\section{Introduction}

One of the most successful realization of semiconductor quantum wires is obtained by growing two quantum wells along perpendicular crystallographic directions. The electronic state in the region of the intersection of the two quantum wells has its motion quantized along two directions while it is free along the intersection line. Such structure is called T-wire and the quantum wells are referred to as arm and stem quantum wells in respect to the $\mathrm{T}$ shape of the system.

In this work we calculate the optical absorption spectra of a semiconductor T-wires in presence of a magnetic field, applied perpendicularly to the plane of the arm quantum well. It is used to externally to enhance the confinement of the eletron-hole pair in the wire and consequently to produce an energy shift of the fundamental excitonic transition [1].

Bryant and Band[2] has performed a calculation using a model where the excitonic wavefunction was written as a product of the T-wire eigenstates for the electron and hole and a variational wavefunction for the e-h correlated state. The trial wavefunction that better reproduced the experimental data was shown to be that in which the e-h correlation was in all three directions. In the work of Goldini et al.[3], a detailed study of the spin mixing in the valence band, neglecting the e-h Coulomb interaction has shown that the character of the hole state (as being heavy or light) depends on the degree of the wavefunction spreading over the arm and stem quantum wells. This has an important effect on the optical anisotropy for light excitation with polarization parallel or perpendicular to the wire axis.

The optical absorption spectrum of a cylindrical quantum wire was recently calculated by Hughes and Citrin[4] who solved the low-density semiconductor Bloch equation (SBE) in the time and real-space domains. The time evolution of the interband optical polarization was obtained by wave packet propagation implemented by split-step method. ¿From the peaks in the spectral response one can find the energies of the electron-hole pair in the wire without having to rely in restrictive basis expansion, perturbation or variational methods.

In this work we study the electroabsorption of magnetoexcitons in a T-shaped quantum wire, under the presence of an electric field is applied along the wire axis. The peak and the linewidth of the optical absorption for the fundamental transition are investigated as functions of the strength of both fields, magnetic and electric. The optical spectra are obtained using an effective 1D potential for the Coulomb attraction between the e-h pair, as a function of the relative e-h distance along the wire, which was calculated using the electron and hole ground states for the laterally confined carriers in a T-wire structure. These states are calculated numerically by solving the 2D Schrödinger equation.

\section{Optical absorption of a T-wire semiconductor}

We begin formulating the problem of an electron and a hole in a T-wire structure neglecting the electron-hole interaction. The ground states we here calculated will be used to obtain the effective Coulomb potential in the wire.

The lateral ground states of the electron in the T-wire satisfy the Schrödinger equation

$$
\left[\frac{1}{2 m_{e}}\left(\mathbf{p}_{\mathbf{e}}-\frac{\mathbf{q}_{\mathbf{e}}}{\mathbf{c}} \mathbf{A}\right)^{2}+V_{2 D}^{e}\right] \phi_{n}^{e}=E_{n}^{e} \phi_{n}^{e},
$$


where $m_{e}$ is the effective mass, $\mathbf{p}_{\mathbf{e}}=-i \hbar \nabla_{\mathbf{e}}$, and $V_{2 D}^{e}$ describes the T-wire potential due to material bandgap differences. This potential is a function of the lateral coordinates, in a cross-section plane of the wire, with periodic boundary conditions. The magnetic field treated Schrödinger equation through a gauge that preserves the translational invariance along the wire, i.e. $\mathbf{A}=\mathbf{A}\left(\mathbf{r}_{\mathbf{e}}\right)=-\mathbf{B} \mathbf{x}_{\mathbf{e}} \widehat{\mathbf{z}}$. The equation for heavy holes is obtained similarly. Spin mixing was not considered in the valence band states calculations, such that a parabolic band approximation for the heavy holes were assumed with anisotropic effective masses $m_{h x}=m_{h z}$ and $m_{h y}$.

The cross term of the square term in the equation above couples the free carrier motion along the wire with the confined motion in the lateral plane (perpendicular) of the wire. As our pair electron-hole are created with zero center-ofmass momentum, the correlation of the e-h relative motion results in momentum distributions for the electrons and holes around the zero momentum, $k_{z}=0$. Therefore we neglect that cross term, and the square term results $\left(\mathbf{p}_{\mathbf{e}}-\frac{\mathbf{q}_{\mathbf{e}}}{\mathbf{c}} \mathbf{A}\right)^{2} \simeq-\hbar^{2} \nabla_{e}^{2}+\left(q_{e} B / c\right)^{2} x_{e}^{2}$. The latter induce a confinement of the electrons and holes around the wire, yielding energy shifts of the electron and hole subbands.

Both Schrödinger equations, for electrons and holes, were solved numerically[5] for the ground-state wavefunctions $\phi_{0}^{e}\left(x_{e}, y_{e}\right)$ and $\phi_{0}^{h}\left(x_{h}, y_{h}\right)$.

\subsection{Optical absorption}

The optical absorption is calculated from the interband polarization whose time evolution is given by the SBE. We consider an optical pulse exciting states around the band gap, such that only the lowest conduction and the highest valence subbands of the wire participate. Coulomb interaction between the electron and hole is then included, and the semiconductor Bloch equation in real-space representation reads $[6,7]$

$$
\begin{array}{r}
i \hbar \partial_{t} P(z, t)=H P(z, t)+d_{0} E(t)[2 n(z, t)-L \delta(z)], \\
i \hbar \partial_{t} n(z, t)=-d_{0} E(t) P^{*}(-z, t)+d_{0}^{*} E^{*}(t) P(z, t),
\end{array}
$$

where

$$
H=-\frac{\hbar^{2}}{2 \mu} \frac{\partial^{2}}{\partial z^{2}}+V(z)+e F z+E_{g}-i \hbar \Gamma,
$$

with $L$ being the wire length, $z=z_{h}-z_{e}$ the relative e-h coordinate along the wire, $F$ an applied longitudinal electric field, $\mu$ the e-h reduced mass $\left(\mu^{-1}=m_{e}^{-1}+m_{h z}^{-1}\right), d_{0}$ the interband dipole moment, $E_{g}$ the bandgap energy (or better, the onset of the continuum of free states in the wire) and $E(t)$ the optical pulse. The relaxation rate $\Gamma$, accounting for scattering processes causing dephasing of the optical polarization, is treated within the relaxation time approximation. The effective potential $V(z)$ is calculated using the ground state wavefunctions obtained in earlier section, that is

$$
V(z)=-e^{2} \int d^{2} \rho_{e} \int d^{2} \rho_{h} \frac{\left|\phi_{0}^{e}\left(\rho_{\mathbf{e}}\right)\right|^{2}\left|\phi_{0}^{h}\left(\rho_{\mathbf{h}}\right)\right|^{2}}{\sqrt{z^{2}+\left(\rho_{\mathbf{e}}-\rho_{\mathbf{h}}\right)^{2}}} .
$$

Equations (2) and (3) describe the space-time evolution of the interband optical polarization $P(z, t)$ and the carrier occupation $n(z, t)$. The effect of this occupation is to bleach the absorption due to phase-space filling. We have not included in the present calculation nonlinear terms in Eqs. (2) and (3) responsible for bandgap renormalization, vertex and local-field corrections. These terms are important when treating high-density, or high excitation situations.

\section{Results and discussion}

We have investigated a T-wire system made of $\mathrm{GaAs} / \mathrm{Al}_{0.3} \mathrm{Ga}_{0.7} \mathrm{As}$ semiconductors for which the effective mass for electron is $m_{e}=0.067 m_{0}$ and heavy hole are $m_{h x}=m_{h z}=0.13 m_{0}$ and $m_{h y}=0.34 m_{0}$, with $m_{0}$ being the free electron mass. The effective masses are considered to be the same for the well and barrier materials. The potential barriers, due to bandgap energy difference of such materials, are for the conduction electron $V^{e}=219.7 \mathrm{meV}$ and for the valence heavy hole $V^{h}=134.6 \mathrm{meV}$. The dielectric constant used is $\varepsilon_{b g}=13.1 \varepsilon_{0}$. The investigated system is similar to previously studied experimentally [1] and theoretically [2]. It has an arm quantum well of width $5.6 \mathrm{~nm}$ and a stem quantum well of width $5.1 \mathrm{~nm}$.

We investigate also the effect of a static electric field applied along the wire axis. The electron and hole in the exciton is pulled apart by the field introducing an additional potential $e F z$ to the effective Coulomb potential $V(z)$, which has no e-h bound eigenstate associated with it. Here we focus our attention only on the fundamental exciton transition.

In Fig.(a) we plotted the absorption peak, relative to the continuum onset, for different applied magnetic fields as a function of the electrical field. The binding energy enhances slightly and then recovers. The inset of Fig.(a) shows the optical absorption considering $B=4 \mathrm{~T}$, for $F=0$ (dotted line) and for $F=4.5 \mathrm{kV} / \mathrm{cm}$ (solid line). We see that the effects of the static electric field are (i) to broaden the fundamental exciton peak, (ii) to shift the peak, and (iii) to produce the characteristic Franz-Keldysh oscillations in the continuum part of the spectrum. In Fig.(b), we observe that the broadening of the fundamental line increase with magnetic field. The linewidth broadening is related to the reduction of the lifetime of the exciton state, which becomes unstable due to the electric field. The inset Fig.(b) shows that the different curves of Fig.(b) can be fitted essencially in the same one when plotted as a function of a convenient scaled electrical field $F^{*}[6]$ which is related to the field necessary to ionize the exciton.

So far we have not considered the carriers population, $n(z, t)=0$, in the SBE, i.e. the bleaching effect. It is necessary to solve Eqs. (2) and (3) together. In this case, the absorption is dependent on the intensity of the optical pulse $E_{0}$. The bleach of the absorption due to phase-space filling 
occurs, and a negative absorption is observed in the case of strong optical pump [6].

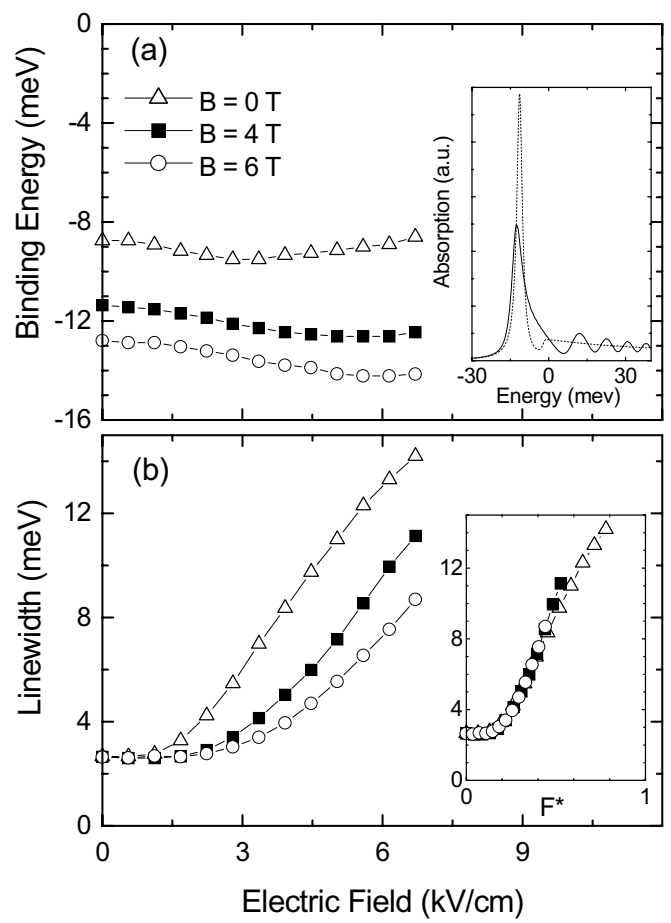

Figure 1. (a) Peak position relative to the onset of the wire continuum and (b) linewidth (FWHM) of the fundamental magnetoexciton transition from the absorption spectra as a function of the electric field applied along the wire for different values of magnetic field. Insets: (a) Absorption spectra at $\mathrm{B}=4 \mathrm{~T}$, without (dashed line) and with (solid line) an applied electric field $F=4.5 \mathrm{kV} / \mathrm{cm}$. (b) Linewidths as function of the electric field scaled by the exciton binding energy $\mathrm{E}_{0}$ and Bohr radius $\mathrm{R}$ (i.e., $F^{*} \sim F R / E_{0}$ ).
In conclusion, we have investigated the fundamental magnetoexciton state in T-wire semiconductor. The effective Coulomb potential was numerically calculated from the ground-state wavefunctions for the electron and hole in the a realistic T-wire. The binding energy and the linewidth of the fundamental magnetoexciton state were obtained from the semiconductor Bloch equation. It was observed an enhancement of the exciton binding energy with the magnetic field strength, [2] and a blue shift of the fundamental transition was seen, as experimentally observed. [1] The linewidth broadening fro different magnetic field strength can be fitted in one curve scalling the electric field by the exciton binding energy and Bohr radius.

\section{Acknowledgments}

We acknowledge support from FAPESP (Brazil).

\section{References}

[1] T. Someya, H. Akiyama, and H. Sakaki, Phys. Rev. Lett. 74, 3664 (1995).

[2] G.W. Bryant and Y.B. Band, Phys. Rev. B 63, 115304 (2001).

[3] G. Goldini, F. Rossi, E. Molinari, and A. Fasolino, Phys. Rev. B 55, 7110 (1997).

[4] S. Hughes and D.S. Citrin, Phys. Rev. Lett. 84, 4228 (2000).

[5] M.H. Degani, Appl. Phys. Lett. 70, 4362 (1991).

[6] J. R. Madureira, M. Z. Maialle, and M.H. Degani, Phys. Rev. B 66, 075332 (2002)

[7] H. Haug and S. W. Koch, Quantum Theory of the Optical and Electronic Properties of Semiconductors, 3rd ed. (World Scientific, Singapore, 1994). 